\title{
Travelling Wave solutions of hyperbolic Telegraph equation by Tanh method
}

\section{Anjali Verma ${ }^{1}$, Amit Verma ${ }^{2}$}

${ }^{1}$ Assistant ProfessorUniversity Centre for Research and DevelopmentChandigarh University, Gharuan, Mohali, Punjab-140413, India

${ }^{2}$ Assistant ProfessorUniversity Centre for Research and DevelopmentChandigarh University, Gharuan, Mohali, Punjab-140413, India

Article History: Received: 11 January 2021; Accepted: 27 February 2021; Published online: 5 April 2021

\begin{abstract}
Tanh method is utilized to find travelling solutions of second order nonlinear Telegraph equation. As a result, we attain dissimilar travelling wave solutions. Our aim is to show that this method is most efficient and convenient approach for verdict travelling wave solutions of nonlinear differential equations. For calculation the software MAPLE is used.
\end{abstract}

KeyWords: Tanh- Method, Travelling wave solutions, (1+1)-dimensional telegraph equation.

\section{Introduction}

Telegraph equation is frequently used in the learning of proliferation of electric signals in a wire conduction stripe and also in the wave phenomenon. The second order nonlinear telegraph equation is:

$$
w_{t t}-w_{x x}+w_{t}+\alpha w+\beta w^{3}=0
$$

Equation of this kind arise in study of heat transfer, chemical kinetics, population dispersal and transmission lines. Various mathematical methods have been planned for obtaining exact and numerical solutions. Mehrdad Lakestani et al. [6] used interpolating scaling functions to find solutions of linear hyperbolic equation. J. Rashidinia et al. [3] used a collocation approach to find solutions of telegraph equation. Murat et al. [4] used DGJ method to find solutions of hyperbolic telegraph equation. A modified adomian decomposition method was used by Hind et al. [2] to find approximate solutions of nonlinear telegraph equation.

T. S. Jang [7] showed how the novel nonlinear telegraph equation can be changed into corresponding scheme of two integral equations of second type. Mustafa et al. [5] Proposed reproducing kernel method to solve telegraph equation with primary conditions used on reproduce kernel theory. A reduced differential transform method was used by Vineet K. Srivastava et al. [8] to solve telegraph equation. So there are many methods to find exact and numerical solutions of nonlinear equations. The method was firstly introduced by Malfliet [9] and Fan and Hon [1].

\section{Methodology}

In this section, a concise depiction of method as follows:

Let us suppose a nonlinear equations in one variable

$$
Q\left(w, w_{x}, w_{t}, w_{x x}, w_{x x x} \ldots \ldots \ldots \ldots\right)=0
$$

Now make the transformation:

$$
w(x, t)=w(\xi), \quad \xi=x-c t
$$

Using this transformation we obtain ODE

$$
Q\left(w, w^{\prime},-c w^{\prime}, w^{\prime \prime}, w^{\prime \prime \prime} \ldots \ldots \ldots \ldots\right)=0
$$

Now set up a new independent variable in the form

$$
K=\tanh (\xi), \quad \xi=x-c t
$$

This leads to the change of derivatives 


$$
\begin{aligned}
& \frac{d}{d \xi}=\left(1-K^{2}\right) \frac{d}{d K} \\
& \frac{d^{2}}{d \xi^{2}}=\left(1-K^{2}\right)\left(-2 K \frac{d}{d K}+\left(1-K^{2}\right) \frac{d^{2}}{d K^{2}}\right) \\
& \frac{d^{3}}{d \xi^{3}}=\left(1-K^{2}\right)\left(\left(6 K^{2}-2\right) \frac{d}{d K}-6 K\left(1-K^{2}\right) \frac{d^{2}}{d K^{2}}+\left(1-K^{2}\right)^{2} \frac{d^{3}}{d K^{3}}\right)
\end{aligned}
$$

Now introduce ansatz

$$
u(\xi)=\sum_{r=-u}^{v} a_{r} K^{r}
$$

Here $\mathrm{u}$ and $\mathrm{v}$ are nonnegative integers. Now put (2.4) and (2.5) into ODE (2.2) .Now to find $\mathrm{u}$ and $\mathrm{v}$ use homogenous balance method and equate coefficients of powers of $\mathrm{k}$ to zero. As a result we find algebraic equations involving $a_{r}$. Solve these equations, and then we get values of parameter. Using (2.5) we obtain exact solutions.

\section{Application of Tanh method}

As described in previous section, we use transformation

$$
w(x, t)=w(\xi), \quad \xi=(x-c t)
$$

1. As a outcome of the first step, we get the nonlinear ordinary differential equation in the Type

$$
\begin{gathered}
w(x, t)=w(\xi), \quad \xi=x-c t . \text { Then we get } \\
-c^{2} w^{\prime \prime}-w^{\prime \prime}-c w^{\prime}+\alpha w+\beta w^{3}=0
\end{gathered}
$$

By balancing the nonlinear terms we get $u=v=1$

Now choose the solutions of (3.3) in the form

$$
w=w(\xi)=a_{-1} K^{-1}+a_{0}+a_{1} K
$$

Where $Y=\tanh (\xi)$. Now put (3.4) into (3.3) and equate like powers of $Y$ equal to zero, then we get following algebraic equations:

$$
\begin{aligned}
& \beta a_{1}^{3}-2 a_{1}-2 c^{2} a_{1}=0 \\
& 3 \beta a_{0} a_{1}^{2}+c a_{1}=0 \\
& 3 \beta a_{0}^{2} a_{1}+3 \beta a_{-1} a_{1}^{2}+2 a_{1}+2 c^{2} a_{1}+\alpha a_{1}=0 \\
& \beta a_{0}^{3}-c a_{1}-c a_{-1}+6 \beta a_{-1} a_{0} a_{1}+\alpha a_{0}=0 \\
& 2 c^{2} a_{-1}+3 \beta a_{-1}^{2} a_{1}+3 \beta a_{-1} a_{0}^{2}+\alpha a_{-1}+2 a_{-1}=0 \\
& 3 \beta a_{-1}^{2} a_{0}+c a_{-1}=0 \\
& -2 a_{-1}-2 c^{2} a_{-1}+\beta a_{-1}^{3}=0
\end{aligned}
$$

Solve the following structure with the support of Maple and get following cases:

1.

$$
a_{0}=a_{0}, a_{1}=0, a_{-1}=0, c=c, \alpha=-\beta a_{0}^{2}, \beta=\beta
$$

2.

$a_{0}=0, a_{1}=0, a_{-1}=a_{-1}, c=0, \alpha=-2, \beta=\frac{2}{a_{-1}^{2}}$ 
3.

$$
\begin{aligned}
a_{-1} & =a_{0}, a_{0}=a_{0}, a_{1}=0, c=\operatorname{RootOf}\left(6+6 Z_{-} Z^{2}+Z\right), \alpha \\
& =\frac{4}{3} \operatorname{RootOf}\left(6+6 \_Z^{2}+{ }_{-} Z\right), \beta= \\
& -\frac{1}{3} \frac{\operatorname{RootOf}\left(6+6 Z^{2}+Z_{Z}\right)}{a_{0}^{2}}
\end{aligned}
$$

4.

$$
\begin{aligned}
a_{0}= & a_{0}, a_{1}=0, a_{-1}=-a_{0}, c=\operatorname{RootOf}\left(6 Z^{2}-Z_{-}+6\right), \alpha= \\
& -\frac{4}{3} \operatorname{RootOf}\left(6 Z^{2}-Z_{-}+6\right), \beta \\
& =\frac{1}{3} \frac{\operatorname{RootOf}\left(6 Z^{2}-Z+6\right)}{a_{0}^{2}}
\end{aligned}
$$

5.

$a_{0}=0, a_{1}=a_{1}, a_{-1}=0, c=0, \alpha=-2, \beta=\frac{2}{a_{1}^{2}}$

6.

$$
\begin{aligned}
a_{0}= & a_{0}, a_{1}=a_{0}, a_{-1}=0, c=\operatorname{RootOf}\left(6+6 Z^{2}+Z_{-}\right), \alpha \\
& =\frac{4}{3} \operatorname{RootOf}\left(6+6 Z^{2}+\_Z\right), \beta= \\
& -\frac{1}{3} \frac{\operatorname{RootOf}\left(6+6 Z^{2}+Z_{2}\right)}{a_{0}^{2}}
\end{aligned}
$$

7.

$$
\begin{aligned}
a_{0}= & a_{0}, a_{1}=-a_{0}, a_{-1}=0, c=\operatorname{Root} O f\left(6 Z^{2}-{ }_{-} Z+6\right), \alpha= \\
& -\frac{4}{3} \operatorname{RootOf}\left(6 \_Z^{2}-{ }_{-} Z+6\right), \beta \\
& =\frac{1}{3} \frac{\operatorname{RootOf}\left(6 Z^{2}-Z Z+6\right)}{a_{0}^{2}}
\end{aligned}
$$

8.

$a_{0}=0, a_{-1}=a_{1}, a_{1}=a_{1}, c=0, \alpha=-8, \beta=\frac{2}{a_{1}^{2}}$

9.

$$
\begin{aligned}
a_{0}= & a_{0}, a_{1}=-\frac{1}{2} a_{0}, a_{-1}=-\frac{1}{2} a_{0}, c=\operatorname{RootOf}\left(12 Z^{2}-Z_{-}+12\right), \\
& \alpha=-\frac{8}{3} \operatorname{RootOf}\left(12 Z^{2}-Z+12\right), \beta \\
& =\frac{2}{3} \frac{\operatorname{RootOf}\left(12 Z^{2}-Z+12\right)}{a_{0}^{2}}
\end{aligned}
$$

10.

$$
\begin{aligned}
a_{0}= & a_{0}, a_{1}=\frac{1}{2} a_{0}, a_{-1}=\frac{1}{2} a_{0}, c=\operatorname{RootOf}\left(12 Z^{2}+Z_{-}+12\right), \alpha \\
& =\frac{8}{3} \operatorname{RootOf}\left(12 Z^{2}+Z_{-}+12\right), \beta= \\
& -\frac{2}{3} \frac{\text { RootOf }\left(12 Z^{2}+Z+12\right)}{a_{0}^{2}}
\end{aligned}
$$


11.

$a_{0}=0, a_{1}=a_{1}, a_{-1}=-a_{1}, c=0, \alpha=4, \beta=\frac{2}{a_{1}^{2}}$

Substituting these results into (3.4), then we get exact solutions. For example in case 10, we have following exact solution

Case 10

$u_{1}=a_{0}+\frac{1}{2} a_{0} \tanh \left(x-\right.$ Rootof $\left.\left(12 \_Z^{2}+{ }_{-} Z+12\right) t\right)+\frac{1}{2} a_{0} \operatorname{coth}\left(x-\right.$ Rootof $\left.\left(12 \_Z^{2}{ }_{+} Z_{2}+12\right) t\right)$

We can choose any value of $a_{0}$. Similarly, we can find other exact solutions.

\section{References}

1. E. Fan and Y.C. Hon, "Generalized Tanh method extended to special types of nonlinear equations". Zeitschrift Fur Naturforschung. Vol.57, 2002, pp. 692-700.

2. Hind Al-Badrani, Sharefah Saleh, H.O. Bakodah and Al-Mazmumy, "Numerical solutions for nonlinear telegraph equation by modified adomian decomposition mehod" Nonlinear analysis and differential equation. Vol. 4(2016) No. 5, pp. 243-257.

3. J. Rashidinia, S. Jamalzadeh and F. Esfahani, " Numerical solutions of one dimensional telegraph equation using cubic B-Spline Collocation method". Journal of Interpolation and Approximate in Scientific Computing. 2014, pp. 1-8.

4. Murat Sari, Abdurrahim Gunay and Gurhan Gurarslan, "A solution to telegraph equation by using DGJ method". International journal of Nonlinear Sciences. Vol. 17(2014) No. 1, pp. 57-66.

5. Mustafa Inc, Ali Akgul and Adem killicman, "Explicit solution of telegraph equation based on reproducing kernel method". Journal of function spaces. Vol. 2012, Article ID 984682.

6. Mehrdad Lakestani and Behzad Nemati, "Numerical solutions of telegraph equation using interpolating scaling functions". Computers and Mathematics with Applications. Vol. 60, 2010, pp. 1964-1972.

7. T.S. Jang, "A new solution procedure for nonlinear telegraph equation". Commun Nonlinear Sci Numer Simulat. Vol. 29(2015), pp. 307-326.

8. 8.Vineet K. Srivastava, Mukesh K. Awasthi, R.K. Chaurasis and M. Tamsir, "The Telegraph equation and its solutions by reduced differential transform method". Modelling and Simulation in Engineering. Vol. 2013, Article ID 746351

9. W. Malfliet, "Solitary wave solutions of nonlinear wave equations". American Journal of Physics, Vol. 60, 1992, pp. 650-654. 